\title{
Multibeam Reflectarray for Transmit Satellite Antennas in Ka Band Using Beam-Squint
}

\author{
Daniel Martinez-de-Rioja, Eduardo Martinez-de-Rioja, Jose A. Encinar
}

\begin{abstract}
This contribution describes a design concept based on beam squint effect in printed reflectarrays that allows to produce multiple contiguous beams at 19.5 and $20 \mathrm{GHz}$, by discriminating in frequency. A $1.6-\mathrm{m}$ reflectarray has been proposed to produce 10 beams separated 0.5 degree using five feeds with frequency reuse. The simulated radiation patterns show a peak gain better than $47.6 \mathrm{dBi}$, with side-lobe levels close to $-22 \mathrm{~dB}$. The proposed concept can be suitable for multiple spot beam satellites in Ka-band.
\end{abstract}

Keywords - reflectarray, beam squint, multibeam antennas, satellite antennas.

\section{INTRODUCTION}

A reflectarray antenna is composed of a planar array of printed elements on a dielectric sheet over a ground plane. The phase of the reflected field on each element can be adjusted to produce a focused or a shaped beam (usually by varying the dimensions of the printed elements) when the antenna is illuminated by a feed [1]. The effect of beam-squint in reflectarray antennas implies that beam direction changes with frequency. This phenomenon has been previously studied by other authors [2], proving that it is mainly caused by a shifting of the focal point at off-center frequencies, rather than other factors, such as the type of reflectarray element or the lattice size used. Furthermore, some methods have been already proposed to reduce or suppress it [3] [4]. In this paper, the authors present a preliminary design of a printed reflectarray antenna to generate multiple closely separated beams with frequency reuse $\left(f_{1}=19.5 \mathrm{GHz}\right.$ and $\left.\mathrm{f}_{2}=20.0 \mathrm{GHz}\right)$, using beam squint to achieve discrimination in frequency. The selected frequencies are in the allocated band for transmission in $\mathrm{Ka}$ band multi-spot beam satellites. The proposed concept will allow a reduction in the number of feeds needed to provide the multi-spot coverage.

\section{BEAM SQUint STUDY}

Beam squint will be exploited by computing the feed position to provide two adjacent beams in the appropriate directions at two relatively close frequencies. Let us consider a reflectarray designed to radiate a focused beam in the direction $\left(\theta_{b}, \varphi_{b}\right)$ at frequency $f_{1}$. Our goal is to modify the pointing direction a small angle $\Delta \theta_{\mathrm{b}}$ at frequency $\mathrm{f}_{2}$ using the same feed. Based on differential spatial phase delay concept, as defined in [1] (pg. 5), and its variation at frequencies $f_{1}$ and $\mathrm{f}_{2}$, an approximate expression can be deduced for the difference of paths from the phase center of the feed to the reflectarray edges (see Fig. 1):

$$
\Delta d=d_{2}-d_{1}=d_{1}^{\prime}+\frac{k_{0} D \cdot \tan \left(\Delta \theta_{b}\right)}{2 \pi \Delta f} \cdot c
$$

where $\mathrm{D}$ is the reflectarray diameter and $\Delta \mathrm{f}=\mathrm{f}_{2}-\mathrm{f}_{1}$. The value of $d_{1}$ ' is equal to zero in case of broadside radiation $\left(\theta_{b}=0^{\circ}\right)$, when the orthogonal plane matches the reflectarray surface. Otherwise, $d_{1}$ ' can be calculated as follows:

$$
d_{1}^{\prime}=D \cdot \sin \left(\theta_{b}\right)
$$

Then, for a fixed value of $d_{1}$, the previous expressions can be used to obtain $\mathrm{d}_{2}$. The coordinates of the feed phase center so that the beam at frequency $f_{2}$ was diverted an angle $\Delta \theta_{b}$ can be found by tracing two circumferences of radius $d_{1}$ and $d_{2}$.

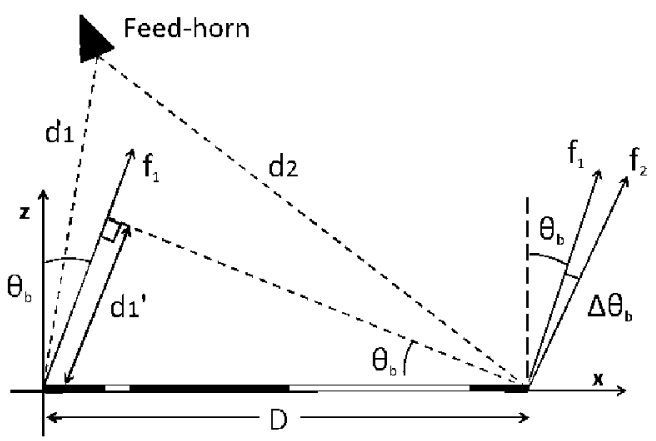

Fig. 1. Reflectarray view for the analysis of beam squint.

\section{REFLECTARRAY DESIGN}

A circular reflectarray, consisting of 31,428 elements arranged in a $200 \times 200$ grid with period $P_{X}=P_{Y}=8 \mathrm{~mm}(160-$ $\mathrm{cm}$ diameter), has been designed to produce two focused beams in the directions $\theta_{\mathrm{bl}}=5^{\circ}, \varphi_{\mathrm{bl}}=0^{\circ}$ at $\mathrm{f}_{1}=19.5 \mathrm{GHz}$, and $\theta_{\mathrm{b} 2}=5.5^{\circ}, \varphi_{\mathrm{b} 2}=0^{\circ}$ at $\mathrm{f}_{2}=20 \mathrm{GHz}$. The antenna is illuminated by a feed-horn, whose phase center is placed at coordinates $\mathrm{x}_{\mathrm{F}}=-109 \mathrm{~cm}, \mathrm{y}_{\mathrm{F}}=0 \mathrm{~cm}, \mathrm{z}_{\mathrm{F}}=218 \mathrm{~cm}$ with respect to the reflectarray center (the feed position has been obtained as 
explained in the previous section). The field radiated by the horn is modelled using a $\cos ^{\mathrm{q}}(\theta)$ distribution, with $\mathrm{q}=13$ for 19-20 $\mathrm{GHz}$ band, which provides $-12 \mathrm{~dB}$ edge illumination. The required phase-shift to be introduced by each reflectarray element can be calculated using the expression provided in [1] (pg. 34), considering for the design the values of $\theta_{b}=5.25^{\circ}$, $\varphi_{b}=0^{\circ}$ at $19.75 \mathrm{GHz}$, in order to reduce the distortion in the radiation patterns at frequencies $f_{1}$ and $f_{2}$. The resulting phaseshift can be accomplished by adjusting the dimensions of the printed elements, employing variable size patches or parallel dipoles [5], but ideal reflectarray cells providing the same phase-shift at $f_{1}$ and $f_{2}$ are assumed in the following results.

In addition to the single-fed reflectarray, a multibeam reflectarray has been designed and simulated. Once the phase distribution has been defined for the initial feed, four additional feed positions have been found to generate beams separated $1^{\circ}$ respect to the central feed. Note that each feed radiates two beams, and therefore the antenna will produce 10 beams in the XZ plane with $0.5^{\circ}$ separation, employing 5 feeds that radiate at $19.5 \mathrm{GHz}$ and $20 \mathrm{GHz}$.

\section{Simulation RESUltS}

The simulated radiation patterns in gain $(\mathrm{dBi})$ in the elevation plane (XZ) for the single-fed reflectarray, shown in Fig. 2, have been obtained from the tangential reflected field at each reflectarray element. The beams are focused in the directions $\theta_{b}=5^{\circ}, \varphi_{b}=0^{\circ}$ at $19.5 \mathrm{GHz}$ and $\theta_{b}=5.5^{\circ}, \varphi_{b}=0^{\circ}$ at 20 $\mathrm{GHz}$, as it was intended $\mathrm{A}$ gain of $49.1 \mathrm{dBi}$ is reached at 19.5 $\mathrm{GHz}$, and $49.3 \mathrm{dBi}$ gain is attained at $20 \mathrm{GHz}$. The beam width at $-3 \mathrm{~dB}$ is lower than $0.65^{\circ}$, and side-lobe level is close to -22 $\mathrm{dB}$ respect to the maximum.

The position of the 5 feeds and the radiation patterns in the elevation plane that resulted from the multibeam reflectarray design are shown in Fig. 3. The distances between the feeds are: $49.7,50.3,50.9$ and $51.4 \mathrm{~mm}$. The radiation patterns in Fig. 3 b show a gain between $49.3 \mathrm{dBi}$ and $47.6 \mathrm{dBi}$, with low side lobes. The beams near the edge show certain distortion, which can be minimized by optimizing the required phase distribution and the reflectarray cells in a detailed design of the antenna.

\section{CONCLUSIONS}

The results for the conducted simulations are promising, because demonstrate that a single feed can generate adjacent beams at different frequencies in multiple spot beam Ka-band antennas by using beam squint effect. A preliminary design has shown that 10 contiguous beams can be generated in one plane using only 5 feeds with frequency reuse. This concept will be further investigated in the future, as well as their application to the design of multibeam antennas.

\section{ACKNOWLEDGMENT}

This work has been supported by the Spanish Ministry of Economy and Competitiveness under the project TEC201343345-P and by the Regional Government of Madrid under project SPADERADAR-CM (P2013/ICE-3000).

\section{REFERENCES}

[1] J. Huang and J. A. Encinar, "Reflectarray Antennas", IEEE Press/Wiley, Piscataway, New Jersey, 2008.

[2] E. Almajali, D. A. McNamara, J. Shaker, M. R. Chaharmir, "On Beam Squint in Offset-Fed Reflectarray", IEEE Antennas and Wireless Propagation Letters, vol. 11, pp. 937-940, August 2012.

[3] S. D. Targonski and D. M. Pozar, "Minimization of Beam Squint in Microstrip Reflectarrays Using an Offset Feed," IEEE Antennas and Propagation Soc. International Symposium, pp. 1326-1329, July 1996.

[4] E. Almajali, D. A. McNamara, J. Shaker, M. R. Chaharmir, "Beam Squint Suppression in Offset-Fed Reflectarray", IEEE Antennas and Wireless Propagation Letters, vol. 12, pp. 587-590, December 2013.

[5] R. Florencio, J. A. Encinar, R. R. Boix, V. Losada, G. Toso, "Reflectarray Antennas for Dual Polarization and Broadband Telecom Satellite Applications," IEEE Trans. on Antennas and Propag., vol. 63, pp. 1234-1246, April 2015.

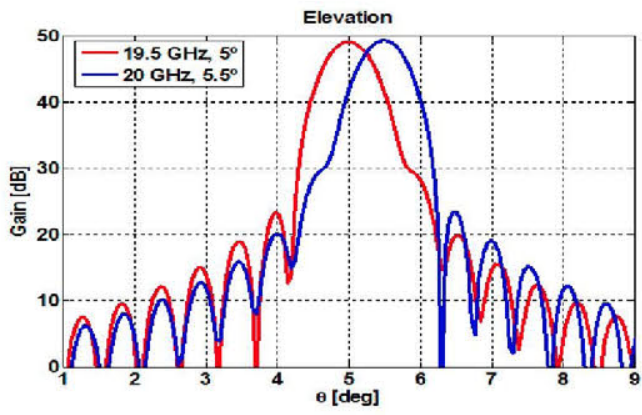

Fig. 2. Radiation patterns in gain $(\mathrm{dBi})$ in the $\mathrm{XZ}$ plane for singlefed reflectarray, with one beam at each frequency.

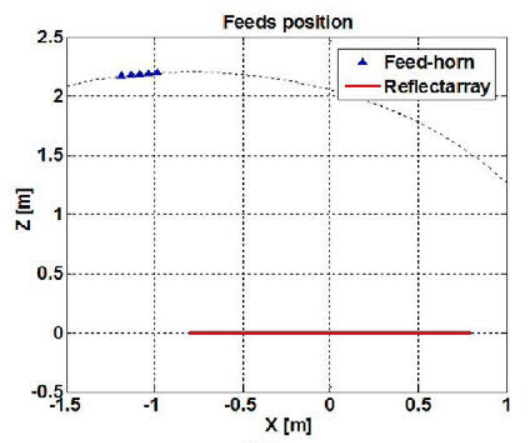

(a)

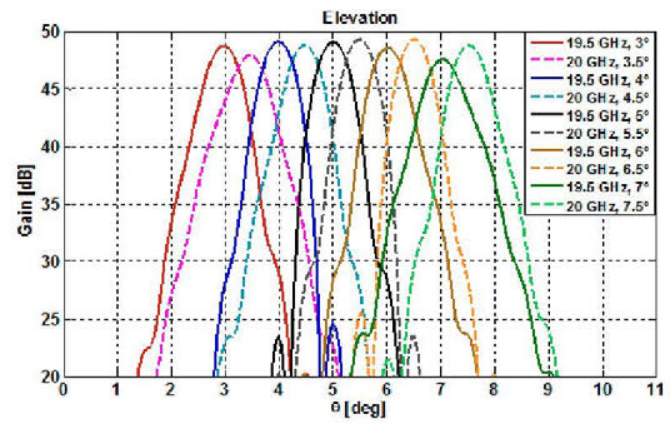

(b)

Fig. 3. Multibeam reflectarray: (a) positions for 5 feeds separated around $5-\mathrm{mm}$, (b) radiation patterns $(\mathrm{dBi})$ in the $\mathrm{XZ}$ plane to generate 10 beams at $19.5 \mathrm{GHz}$ and $20 \mathrm{GHz}$. 